\title{
Introduction to the Special Issue for SPAA 2018-Part 2
}

This issue of the ACM Transactions on Parallel Computing (TOPC) contains selected papers from the 30th ACM Symposium on Parallelism in Algorithms and Architectures (SPAA 2018), which was held July 16-18, 2018, in Vienna, Austria.

We invited several outstanding papers, representing a diverse set of topics in parallel computing, that were particularly well liked by the reviewers and program committee. A previous issue of TOPC included three such papers. This issue includes four more:

1. Gopal Pandurangan, Peter Robinson, and Michele Scquizzato: "On the Distributed Complexity of Large-Scale Graph Computations"

2. Barbara Geissmann and Lukas Gianinazzi: "Parallel Minimum Cuts in Near-linear Work and Low Depth"

3. Giorgio Lucarelli, Benjamin Moseley, Nguyen Kim Thang, Abhinav Srivastav, and Denis Trystram: "Online Non-preemptive Scheduling on Unrelated Machines with Rejections"

4. Kjell Winblad, Konstantinos Sagonas, and Bengt Jonsson: "Lock-free Contention Adapting Search Trees"

We thank the authors for their efforts in producing mature versions of their manuscripts. We would also like to thank the TOPC reviewers as well as the SPAA 2018 program committee members and external reviewers who provided useful feedback for improving these papers.

Jeremy Fineman

Program Chair

Aydin Buluc

Program Committee

Seth Gilbert

Program Committee

Guest Editors

\section{ACM Reference format:}

Jeremy Fineman, Aydin Buluc, and Seth Gilbert. 2021. Introduction to the Special Issue for SPAA 2018-Part 2. ACM Trans. Parallel Comput. 8, 2, Article 6 (July 2021), 1 page.

https://doi.org/10.1145/3463366

(c) 2021 Copyright held by the owner/author(s).

2329-4949/2021/07-ART6

https://doi.org/10.1145/3463366 